\title{
KINOMIC ALTERATIONS IN ATYPICAL MENINGIOMA
}

\author{
Joshua C. Anderson ${ }^{1}$, Robert B. Taylor ${ }^{1}$, John B. Fiveash ${ }^{1}$, Rik de Wijn ${ }^{2}$, G. Yancey \\ Gillespie $^{1}$, Christopher D. Willey ${ }^{1 *}$ \\ Affiliations: ${ }^{1}$ University of Alabama at Birmingham, Department of Radiation Oncology, \\ ${ }^{2}$ PamGene International B.V., 's-Hertogenbosch, The Netherlands
}

\section{Emails:}

JCA = janders211@gmail.com

RBT= $\underline{\text { rbtaylor@uabmc.edu }}$

JBF= jfiveash@uabmc.edu

RDW = rdwijn@ pamgene.com

GYG = yancey@uab.edu

$\mathrm{CDW}=\underline{\text { cwilley@uabmc.edu }}$

Short Title: Kinomics in Atypical Meningioma

\section{*Corresponding author:}

Christopher D. Willey, MD, PhD

HSROC 2232C

Department of Radiation Oncology

The University of Alabama at Birmingham

Birmingham, AL 35249

Phone: 205-934-5670

Email: cwilley@uabmc.edu

The authors disclose no conflicts of interest. 


\begin{abstract}
Background: We sought to profile Atypical Meningioma in a high-throughput manner to better understand the altered signaling within these tumors and specifically the kinases altered in recurrent atypical meningioma. Kinomic Profiles could be used to identify prognostic biomarkers for responders/non-responders to classify future patients that are unlikely to benefit from current therapies. Directly these results could be used to identify drug-actionable kinase targets as well.
\end{abstract}

Methods: Peptide-substrate microarray kinase activity analysis was conducted with a PamStation ${ }^{\circledR} 12$ analyzing the tyrosine kinome in each tumor kinetically against $\sim 144$ target peptides. These data were then analyzed relative to clinical outcome (e.g., tumor recurrence).

Results: 3 major clusters of atypical meningiomas were identified with highly variant peptides primarily being targets of EGFR family, ABL, BRK and BMX kinases. Kinomic analysis of recurrent atypical meningiomas indicated patterns of increased phosphorylation of BMX, TYRO3 and FAK substrates as compared to non-recurrent tumors.

Conclusion: The atypical meningiomas profiled here exhibited molecular sub-clustering that may have phenotypic corollaries predictive of outcome. Recurrent tumors had increases in kinase activity that may predict resistance to current therapies, and may guide selection of directed therapies. Taken together these data further the understanding of kinomic alteration in atypical meningioma, and the processes that may not only mediate recurrence, but additionally may identify kinase targets for intervention.

Keywords: kinomics, kinase activity, personalized medicine, meningioma, radiation 


\section{INTRODUCTION}

Meningiomas are tumors derived from the epithelial cells composing the meningeal layer of the brain and spinal cord. Menigiomas are the most common primary intracranial neoplasm $(\sim 110,000$ cases diagnosed per year) and comprise approximately one third of all primary central nervous system (CNS) tumors. The majority of meningiomas are WHO Grade I, which have excellent prognosis with current treatment, however WHO Grade II (atypical) meningiomas comprise $20 \%$ of all meningiomas by current WHO standards and are difficult to manage with recurrence rates around $40 \%$ after gross tumor resection (Aghi et al., 2009). Those tumors that are recurrent typically have an aggressive course with poor prognosis. However, there is no consensus on the appropriate treatment of atypical meningiomas (Komotar et al., 2012). As in WHO Grade 1 meningiomas, surgery is the primary treatment. Radiation has been shown to improve progression free survival but the appropriate radiation dose and appropriate patient selection have not been identified. The ability to characterize tumors prospectively and identify those patients who would benefit most from radiation or other treatments has not been developed. Moreover, there is little known regarding what factors lead to the subset of meningiomas that become aggressive or recur.

Multiple molecular pathways are likely involved in development, progression, and treatment resistance of meningiomas. Some work has been done in identifying genes associated with meningioma development, much of it centering on losses that converge on the neurofibromatosis-2 (NF2) signaling axis, but to date this work has not resulted in clinically useful targets for therapy or for risk stratification. Kinase driven pathways, including the phosphatidylinositol-3 kinase (PI3K)/Akt/mammalian target of rapamycin (mTOR), and mitogen activated protein kinase (MAPK) pathways are important in cellular survival and proliferation, and are closely tied to NF2 alterations. Formation of meningiomas and progression from lower grade meningiomas can be driven by multiple kinase driven pathways including the receptor tyrosine kinases epidermal growth factor receptor (EGFR), platelet derived growth factor receptor (PDGFR), and insulin-like growth factor receptor (IGFR), as well as losses in cyclin-dependent kinase inhibitor 2A (CDK2NA or p16), and gains in ribosomal protein S6 kinase beta-1 (S6K) associated with higher-grade anaplastic meningiomas (Bostrom et al., 2001; Choy et al., 2011; James et al., 2009; Mawrin et al., 2005).

$$
\text { Despite the improved }
$$

understanding of signaling pathways in this disease, identification of biomarkers and therapeutic targeting of these molecular targets for overcoming therapy resistance has not been successfully translated into the clinic. Issues arise multifold when identifying, primarily at the gene-level, biomarkers for atypical meningioma recurrences such as: 1) passenger mutations that occur and may correlate, but not drive tumor processes; 2) identified genes may not be easily therapeutically targetable at the genetic level; 3) alterations at the genetic level may not directly lead to protein expression level changes, and furthermore; 4) even changes in protein level may not translate into increased enzymatic activity. Indeed, recent development of therapeutic agents 
in oncology has been predominantly focused on targeting kinases through small molecule and antibody based approaches (Blanc, Geney, \& Menet, 2013; Fang, Grutter, \& Rauh, 2013; Powers \& Palecek, 2012; Wang, Zorn, \& Kuriyan, 2014). It follows that kinases, that are predominantly regulated posttranslationally, and inhibition of them, would benefit from being studied directly at their level of enzymatic action - the level that most targeted agents intervene.

Direct measurement of global kinase (kinomic) activity in a high throughput manner allows direct measurement of kinase activity in real time in complex lysates, including primary meningioma samples. We, and others, have used kinomic activity profiling to identify alterations in kinase activity in tumor and other pathologic tissue, including directlyfrom-patient derived tissue (Anderson, Duarte, et al., 2014; Anderson, Minnich, et al., 2014; Duverger et al., 2013; Sikkema et al., 2009). Herein, we describe the kinomic signaling diversity of atypical meningiomas treated at our institution and the kinomics seen specifically in recurrent tumors.

\section{METHODOLOGY}

\subsection{Ethics}

This study was conducted in accordance with institutional policies and was approved by University of Alabama at Birmingham (UAB) Institutional Review Board ("Kinomics of Atypical Meningioma" protocol \# X130215007). Following IRB approval, we conducted this retrospective study. Samples were deidentified prior to kinomic profiling in compliance with HIPAA rules and regulations.

\subsection{Patient Characteristics}

Patient inclusion criteria included those patients 19 years of age or older with a diagnosis of meningioma who had undergone surgery and/or radiation therapy at UAB. Thirty-one samples were acquired from atypical meningioma patients as listed in Table 1. All samples were collected surgically and were flashfrozen in liquid nitrogen prior to transfer to the UAB Brain Tumor Tissue Core (Directed by Dr. Gillespie). These samples were collected between 1999 and 2013. Known atypical meningiomas with frozen tissue were identified and transferred to the UAB Kinome Core in liquid nitrogen for processing and profiling as described below. Further analysis was conducted on a subset of these samples that passed annotation and signal quality control (QC). Of these analyzed, there were 54\% (7) female and $46 \%$ (6) male with a mean age of 54.7 and with $30.8 \%$ (4/13) having recurrent tumors.

\subsection{Kinomic Profiling}

Kinomic profiling was performed at the UAB Kinome Core (www.kinomecore.com). Frozen tissue for each tumor was processed at $4{ }^{\circ} \mathrm{C}$ for kinomic profiling and lysed in M-PER lysis buffer with protease and phosphatase inhibitors per our standard protocol (Anderson, Duarte, et al., 2014). Protein quantitation was performed using standard BCA reaction assay (Pierce Scientific, Waltham, MA). Sixteen $\mu \mathrm{g}$ of protein per tumor were combined with kinase buffer, ATP, and fluorescently labeled anti-PY20 antibodies per UAB Kinome Core standard operating procedure and loaded into each well of the tyrosine (PTK) PamChips ${ }^{\circledR}$. 
The PamStation ${ }^{\circledR} 12$ kinomics workstation (PamGene ${ }^{\circledR} \quad$ International, 'sHertogenbosch, The Netherlands) using the standard PTK PamChip ${ }^{\circledR}$ protocol using Evolve2 Software (v. 1.5) as previously published (Anderson, Duarte, et al., 2014).
As lysates were pumped through the array, images were captured using Evolve2 followed by primary data processing, phosphorylation curve fitting, and quantitation using BioNavigator v. 6.0 (PamGene).

Table 1. Patient Sample Characteristics

\begin{tabular}{|c|c|c|c|c|c|}
\hline ID & Rec Status & Age & Race & Gender & Kinomics \\
\hline M1 & Primary & 73 & Albino & Male & NO SIG \\
\hline M2 & Primary & 50 & Black & Male & LOW SIG \\
\hline M3 & Primary & 73 & White & Female & Yes \\
\hline M4 & Primary & 46 & Black & Male & Yes \\
\hline M5 & Primary & 61 & Black & Male & Yes \\
\hline M6 & Primary & 61 & White & Female & LOW SIG \\
\hline M7 & Recurrent & 70 & White & Female & Yes \\
\hline M8 & Recurrent & 40 & White & Male & LOW SIG \\
\hline M9 & Primary & 59 & Black & Male & Yes \\
\hline M10 & Recurrent & 53 & White & Female & LOW SIG \\
\hline M11 & Unknown & 35 & White & Female & MISMATCH \\
\hline M12 & Unknown & 41 & Black & Male & NO SIG \\
\hline M13 & Primary & 51 & Black & Female & LOW SIG \\
\hline M14 & Unknown & 54 & White & Female & ARRAY ERR \\
\hline M15 & Recurrent & 67 & White & Female & Yes \\
\hline M16 & Recurrent & 39 & White & Female & Yes \\
\hline M17 & Primary & 65 & White & Male & LOW SIG \\
\hline M18 & Primary & 17 & Black & Male & LOW SIG \\
\hline M19 & Recurrent & 48 & White & Male & LOW SIG \\
\hline M20 & Primary & 59 & White & Female & NO SIG \\
\hline M21 & Unknown & 49 & Black & Male & MISMATCH \\
\hline M22 & Recurrent & 47 & Black & Male & MISSING \\
\hline M23 & Primary & 69 & White & Female & NO SIG \\
\hline M24 & Primary & 52 & Black & Female & NO SIG \\
\hline M25 & Recurrent & 69 & Black & Female & Yes \\
\hline M26 & Primary & 40 & White & Male & Yes \\
\hline M27 & Primary & 66 & White & Male & Yes \\
\hline M28 & Unknown & 50 & Black & Female & NO SIG \\
\hline M29 & Primary & 43 & White & Female & Yes \\
\hline M30 & Primary & 30 & White & Female & Yes \\
\hline
\end{tabular}

NO SIG=no signal; LOW SIG=Low signal; ARRAY ERR= array error 
2.4 Data Analysis

Briefly, signal over multiple exposure times was linearly integrated and the slope was $\log 2$ transformed as described previously for heatmap displays (Anderson, Minnich, et al., 2014). Unsupervised hierarchical Euclidean distance metrics and complete linkage clustering of peptide signal across all, or a variance filtered subset, of the 144 substrates for the 13 samples was done using a Clustering PamApp in BioNavigator. Variant peptides were mapped independent of directionality for upstream kinases using a modified query of the Kinexus database, that scored each kinase based on the percentage of times it occurred (in a phosphorylatable-residuecorrected manner) in the top 10 list for each phosphorylatable peptide as before (Anderson, Duarte, et al., 2014). A ranking of putative kinases responsible for changes in phosphorylation activity between recurrent and non-recurrent tumors was done using the Upstream Kinase Analysis PamApp (v8, PamGene). With this tool individual kinases are represented by sets of peptides that are predicted to be phosphorylated by these kinases based on the Kinexus database (www.phosphonet.ca). Kinases were ranked by the significance of the overall effect size and the specificity of the corresponding peptide sets. Identified upstream kinases were uploaded by Uniprot ID's to GeneGo MetaCore (www.portal.genego.com, Thompson Reuters) for network modeling and pathway analysis.

\section{RESULTS}

\subsection{Sample Quality}

Thirty-one surgically collected and flash-frozen samples were identified from the UAB Brain Tumor Tissue Core as potential atypical meningioma patient samples with flash frozen material available. Of these, 4 had inadequate annotation or were duplicated samples, and the remaining atypical meningioma samples were run on the PTK array. Initial analysis revealed that 14 of these had unacceptably low overall array signal (mean peptide signal $<100$ at final prewash cycle). This large percentage of poorsignal samples is potentially due in part to extended collection time at $>4 \mathrm{C}$, quality of tissues collected (i.e. connective tissue/fibrosis/necrosis or other relatively kinase-inert material), or inadequate storage or transport conditions based on our previous experience with primary tumor tissues, although we did not investigate this further (Anderson, Minnich, et al., 2014). Kinase activity can be very sensitive to time and temperature variations during collection, especially prior to snap freezing of tissue. As these tissues were not collected prospectively for kinomic profiling, these conditions were not controlled or recorded. After sample exclusion, there were 13 atypical meningioma samples (4 recurrent and 9 primary tumors) retained for comparative kinomic profiling and analysis.

\subsection{Molecular Clusters of Atypical Meningioma}

The kinomic activity profiles for the 13 evaluable tumors were analyzed using an unsupervised hierarchical clustering algorithm and displayed as a heatmap. Broadly, the dendrogram revealed 3 major subgroup clusters (Figure $\mathbf{1}$, cut at the horizontal red line into groups 'A','B', and 'C'). However, none of these groups were enriched for recurrent tumors. Because many of the phosphopeptide probes in the kinomic profiles did not 
differ among the 13 tumors, we performed a variance filtration step prior to the hierarchical clustering using a variance cutoff of >2.0. As shown in Figure 2A, this simplified clustered heatmap (with phosphopeptide probes indicated) reduced the number of subgroups to 2 with recurrent tumors all falling in group 1. Upstream kinase predictions for these phosphopeptides with network modeling of these kinases in a Direct Interactions Network are shown in Figure 2B and 2C, respectively.

\section{Figure 1.}

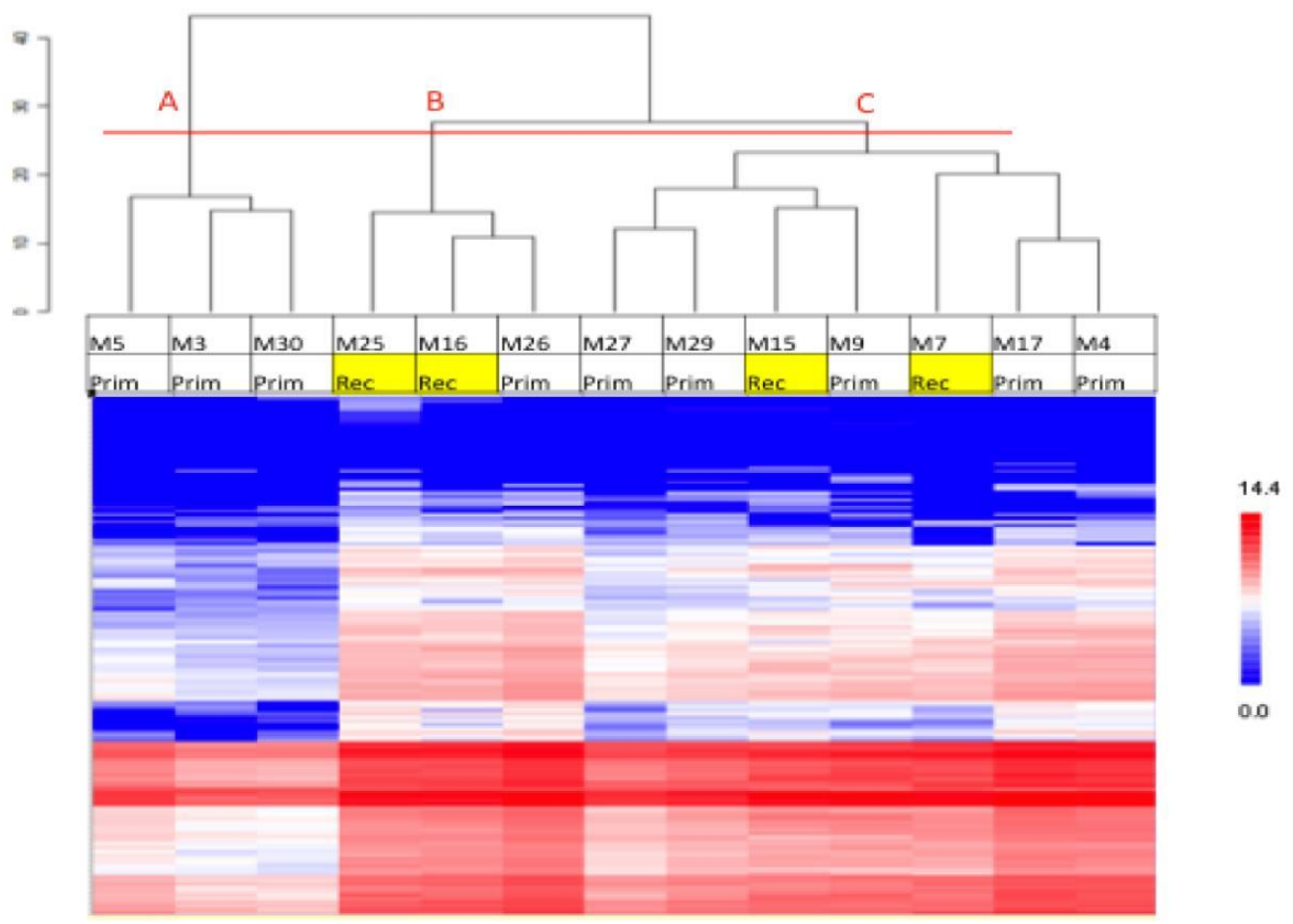

Heatmap of peptide phosphorylation per each meningioma sample. Red indicates a higher signal peptide, and blue indicates lower. Samples (columns on the $\mathrm{x}$ axis) and peptides are clustered on the y axis via dendrogram with recurrent tumors indicated with yellow background. A horizontal red line was added to separate the 3 major clusters of molecular subtypes.

\subsection{Alterations in Recurrent Atypical Meningioma}

Although the variance filtration and subsequent unsupervised hierarchical clustering did group the recurrent atypical meningioma samples into a one major subgroup, several non-recurrent atypical meningiomas also clustered into that subgroup. Therefore, to better elucidate the kinase signaling that is enriched only in the recurrent atypical meningiomas required a more supervised approach. As such, we used a broad whole-array-centric (as opposed to substrate/peptide-centric) approach utilizing PamGene's PTK Upstream Kinase Prediction PamApp. This application uses a Normalized Kinase Score (NKS, sensitivity) and specificity scoring to determine alterations across multiple increased substrates that are 'enriched' for increased kinase activity as compared to decreased activity. We used 
this approach to compare whole array profiles of the 4 recurrent tumors as compared to the 9 non-recurrent tumors. Kinases that were enriched with this method across the tyrosine kinome included bone marrow kinase on $\mathrm{X}$ chromosome (BMX), focal adhesion kinase (FAK), and tyrosine-protein kinase receptor TYRO3 (TYRO3) that were ranked as increased in both specificity and normalized kinase statistic (Figure 3A) in recurrent as compared to the non-recurrent atypical meningiomas.

These kinases were also mapped to a biological network using MetaCore to identify the shortest paths network as shown in Figure 3B. Network mapping identified potential intermediaries such as ETS1, Androgen Receptor, and cyclin dependent kinase 1 (CDK1) along with Src family members that may also be important in the signaling differences seen in recurrent tumors.

\section{Figure 2.}

A. Clustered Peptides

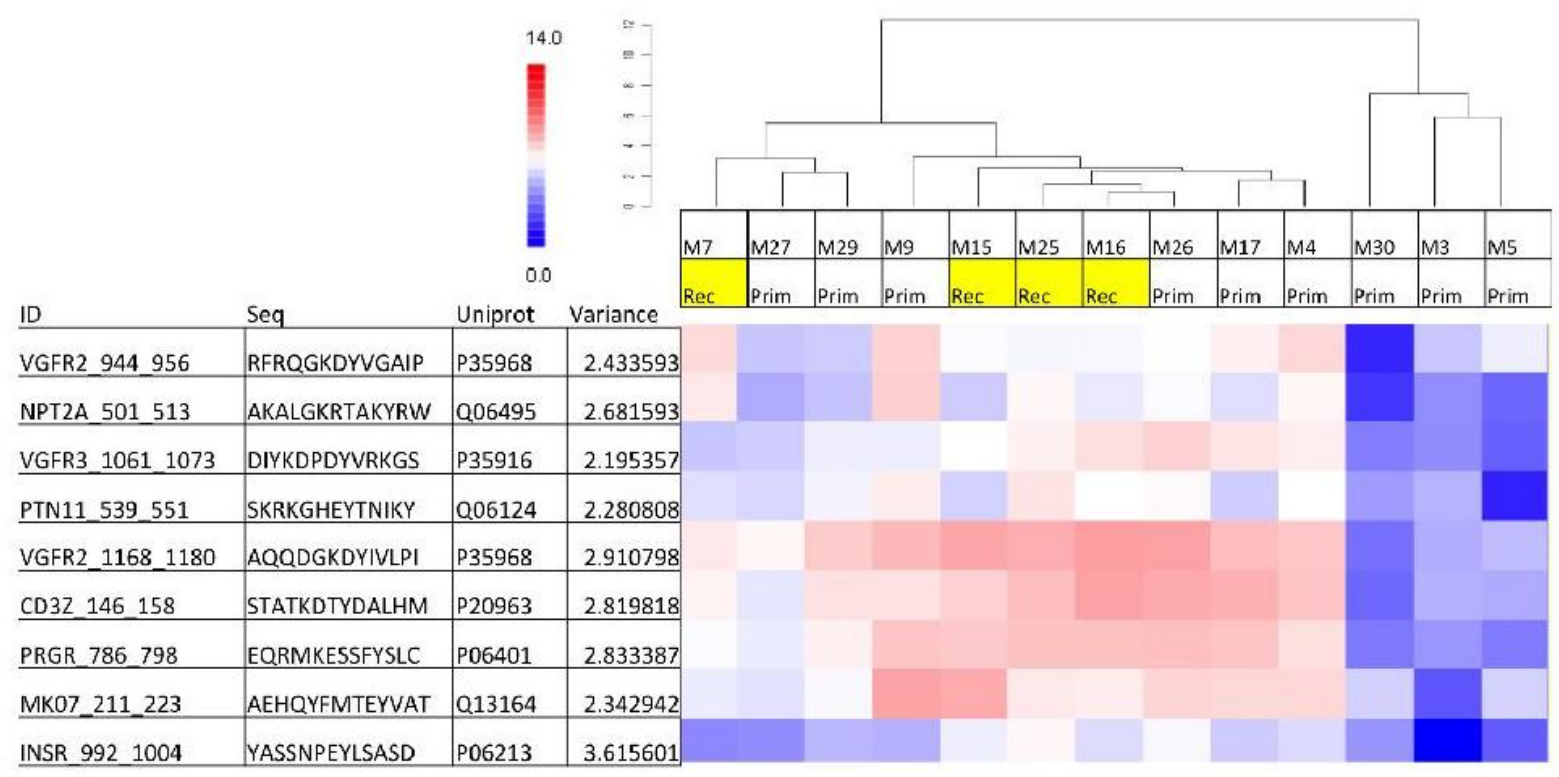

B. Upstream Varian Kinases

\begin{tabular}{|c|c|c|c|c|}
\multicolumn{1}{c}{ kinase } & \multicolumn{1}{c}{ uniprot } & \multicolumn{1}{c|}{ score } & \multicolumn{1}{c|}{ hits } & \%hits \\
\hline CSK & P41240 & 4958 & 8.5 & 47.2 \\
\hline Ab11 & P00519 & 2864 & 7 & 38.9 \\
\hline BRK & Q13882 & 4160.5 & 6.5 & 36.1 \\
\hline ITK & Q08881 & 2667 & 6.5 & 36.1 \\
\hline Arg & P42684 & 2515 & 6 & 33.3 \\
\hline CTK & P42679 & 2387 & 6 & 33.3 \\
\hline EGFR & P00533 & 2240 & 6 & 33.3 \\
\hline ErbB3 & P21860 & 4013.5 & 5.5 & 30.6 \\
\hline Syk & P43405 & 2979.5 & 5 & 27.8 \\
\hline Bmx & P51813 & 2125 & 5 & 27.8 \\
\hline Axl & P30530 & 1608.5 & 5 & 27.8 \\
\hline
\end{tabular}

C. Variant Kinases Network

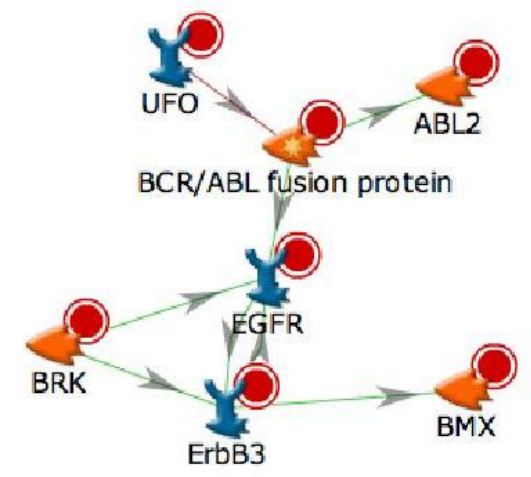


Highly Variant Peptides and Kinases: (A) displays highly variant peptides clustered by sample and a heatmap colored by $\log 2$ signal as relatively high (red) or low (blue). (B) Displays kinases upstream of these peptides ranked based on score that were mapped to (C) a Direct Interactions Network mapping with orphan nodes excluded. Arrowheads denote direction and colors denote positive (green) or negative (red) interactions.

\section{Figure 3.}

A. Kinase Scoring and Specificity

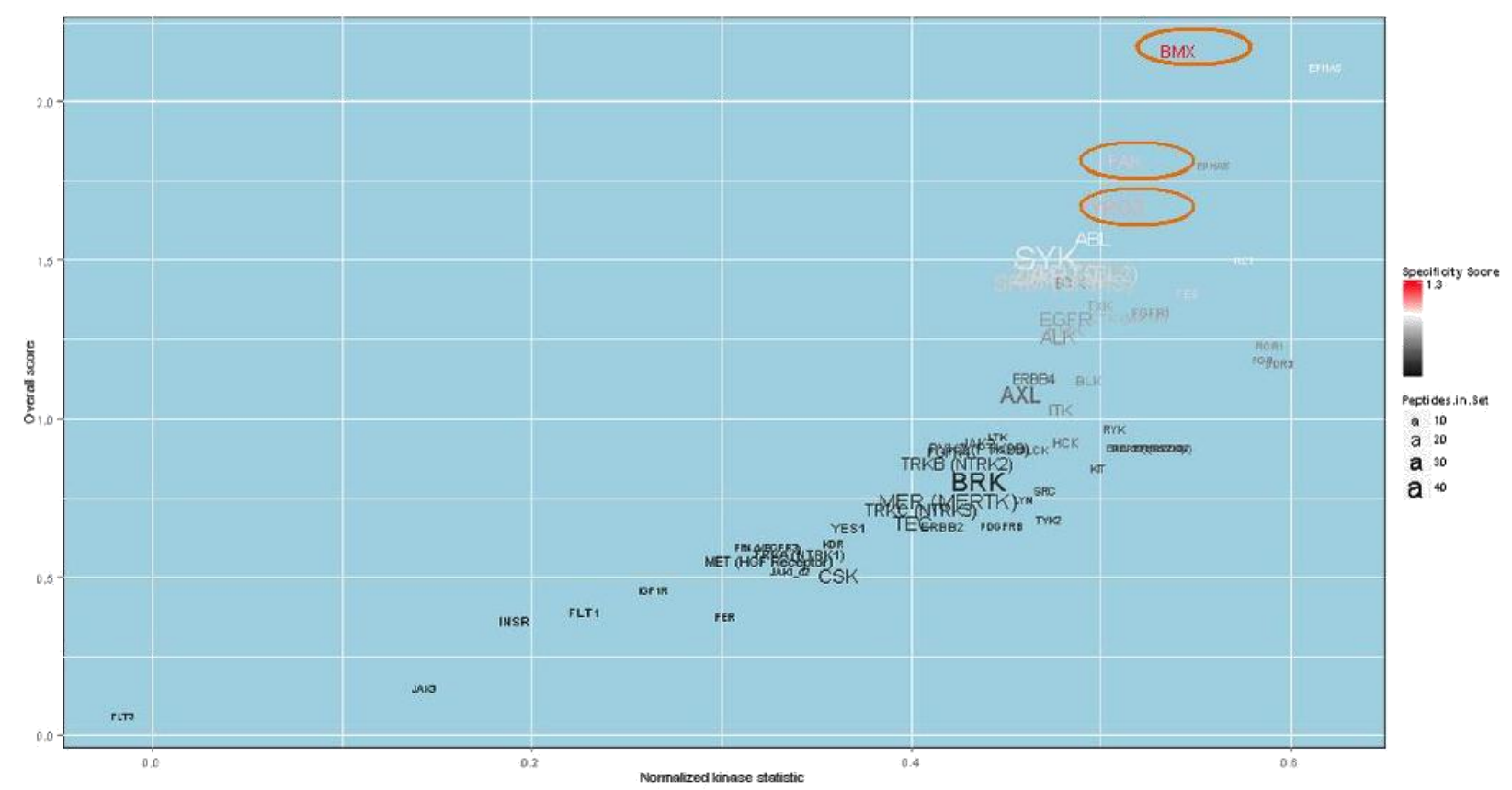

B. Recurrent Kinase Mapping

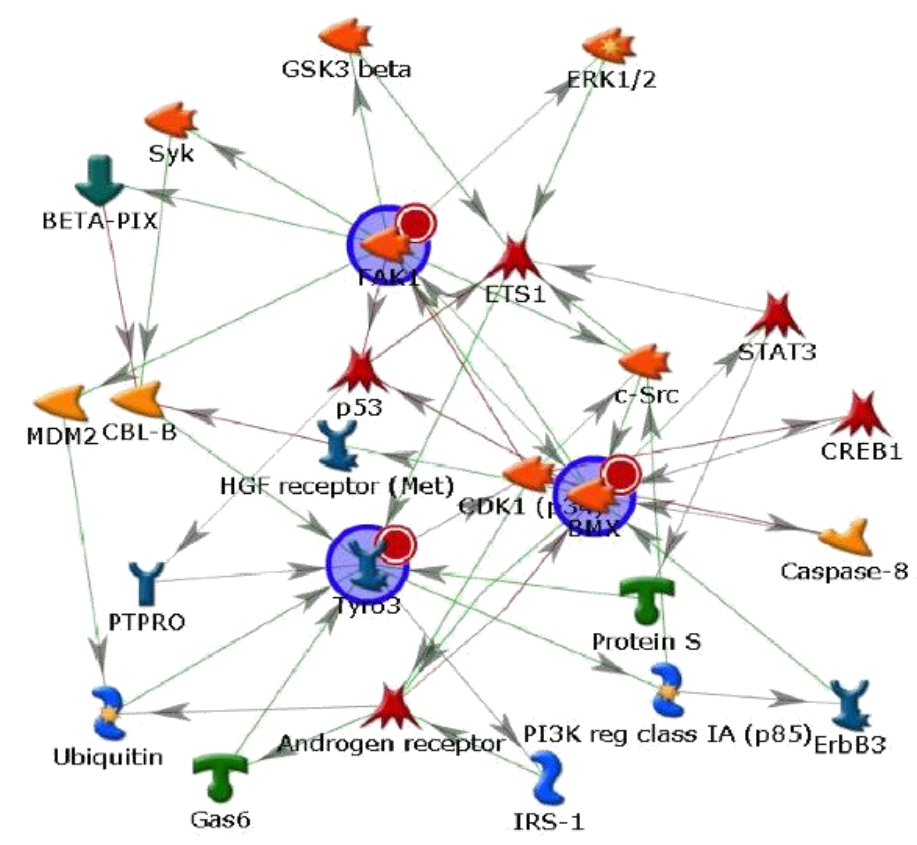


Recurrent atypical meningioma kinomic alterations (increases) relative to primary atypical meningioma were analyzed. (A) Kinases are scored along the $\mathrm{x}$ axis by Normalized Kinase Statistic, and colored by Specificity plotted against overall score on the $y$ axis using the Upstream Kinase Prediction PamApp. Size of kinase text denotes number of peptides targeted by that kinase. (B) Displays a Shortest Paths Mapping for kinases identified as increased in recurrent atypical meningioma. Arrowheads denote direction and colors denote positive (green), or negative (red) interactions.

\section{DISCUSSION}

Currently, the management of atypical meningiomas relies on clinicopathological markers for decisionmaking. The Simpson Grade, described almost 50 years ago (Simpson, 1957), is still used to characterize extent of resection and is somewhat prognostic (Jenkinson et al., 2015). Adjuvant radiation treatment remains controversial in that there are advocates ranging from those promoting early post-operative radiation to those that propose no radiation following gross tumor resection. Most studies to investigate this controversy have been retrospective in nature and have suffered from the expected biases. However, even in the setting of subtotal resection where the adjuvant radiation therapy is more generally expected, the optimal dose and targeting is less clear. Indeed, recently completed prospective phase II clinical trials by the Radiation Therapy Oncology Group (RTOG 0539), the European Organisation for Research and Treatment of Cancer (EORTC 22042-26042) as well as the randomized phase III "Radiotherapy versus Observation following surgical resection of Atypical Meningioma" (ROAM/EORTC 1308) trial will hopefully improve the understanding and proper use of radiation in atypical meningioma. However, systemic therapy options are severely limited. For many years, chemotherapeutics have been tried and systematically failed at changing the clinical management of meningiomas (Jenkinson et al., 2015; Norden, Drappatz, $\&$ Wen, 2007). There has been a steady increase in the understanding of molecular pathobiology of meningiomas, but identifying molecular determinants of aggressiveness/prognosis have been elusive. For example, identifying activating mutations in known oncogenic kinases such as EGFR, KRAS, BRAF, and PIK3CA has revealed that these are relatively rare (Bujko et al., 2014). As such, we sought to explore atypical meningioma kinase activation in a more global fashion and at the level of enzymatic activity.

In our study, we investigated the kinase signaling pathways activated within a cohort of atypical meningiomas using a novel kinomic profiling approach. We further identified potential kinase targets that may be enriched in tumors that behave more aggressively. Specifically, FAK and BMX represent kinases that have been implicated in highly invasive tumors, including glioblastoma multiforme. FAK is a non-receptor tyrosine kinase that can impact many cancer-associated processes ranging from modulation of c-Src and p53 signaling to cancer invasion/migration and is actively being developed clinically (Golubovskaya, 2014) BMX is another non-receptor tyrosine kinase being 
developed as a molecular target in various cancers including other brain tumors, and interestingly may be impacted by small molecule inhibitors of EGFR (Jarboe, Dutta, Velu, \& Willey, 2013). TYRO3 is a member of a family of kinases that may promote survival and play a role in drug resistance and cell migration (Graham, DeRyckere, Davies, \& Earp, 2014). It is interesting to note that we did not identify VEGFR or PDGFR as being highly enriched in recurrent vs. non-recurrent tumors. There has been considerable interest in both receptor tyrosine kinases as molecular targets in aggressive meningiomas, but efficacy data is quite limited. A recent single arm phase II trial was published using sunitinib, a multitargeted tyrosine kinase inhibitor including VEGFR and PDGFR, in the setting of recurrent and progressive atypical and anaplastic meningiomas. While the drug was efficacious with a progression free survival at 6 months of $42 \%$, there was considerable toxicity with $60 \%$ grade 3 or higher toxicity including 1 fatal CNS hemorrhage (Kaley et al., 2015). Because of the potential risk, it is important to carefully select patients that will most benefit from this type of therapy, further supporting the development of molecular biomarkers. Multi-institutional collaborative translational studies will be required to generate this important molecular information as most studies have relied on small patient numbers.

\section{CONCLUSION}

Taken together, kinomic profiling of atypical meningiomas has identified possible molecular subsets across these tumors as well as specific kinases that may be altered in the recurrent subset. In the future, these profiles of phosphosubstrates may help provide biomarkers for predicting tumors that may recur after surgery and radiotherapy. Larger sample sizes are needed to further develop molecular markers.

\section{ACKNOWLEDGEMENTS}

We would like to thank Faris Naji from PamGene for ongoing work on BioNavigator and the associated PamApps. We would also like to thank Christine Duarte from Maine Medical Center Research Institute for her work on the upstream kinase prediction tools. This work was supported in part by the UAB Radiation Oncology Intramural Pilot Grant (RBT and CDW). The Brain Tumor Tissue Core is supported by P50CA097247 and P20CA151129. 


\section{REFERENCES}

Aghi, M. K., Carter, B. S., Cosgrove, G. R., Ojemann, R. G., Amin-Hanjani, S., Martuza, R. L., . . . Barker, F. G., 2nd. (2009). Long-term recurrence rates of atypical meningiomas after gross total resection with or without postoperative adjuvant radiation. Neurosurgery, 64(1), 56-60; discussion $60 . \quad$ doi: 10.1227/01.NEU.0000330399.55586.63

Anderson, J. C., Duarte, C. W., Welaya, K., Rohrbach, T. D., Bredel, M., Yang, E. S., . . . Willey, C. D. (2014). Kinomic exploration of temozolomide and radiation resistance in Glioblastoma multiforme xenolines. Radiother Oncol, 111(3), 468-474. doi: 10.1016/j.radonc.2014.04.010

Anderson, J. C., Minnich, D. J., Dobelbower, M. C., Denton, A. J., Dussaq, A. M., Gilbert, A. N., ... Willey, C. D. (2014). Kinomic profiling of electromagnetic navigational bronchoscopy specimens: a new approach for personalized medicine. PLoS One, 9(12), e116388. doi: 10.1371/journal.pone. 0116388

Blanc, J., Geney, R., \& Menet, C. (2013). Type II kinase inhibitors: an opportunity in cancer for rational design. Anticancer Agents Med Chem, 13(5), 731747.

Bostrom, J., Meyer-Puttlitz, B., Wolter, M., Blaschke, B., Weber, R. G., Lichter, P., . . . Reifenberger, G. (2001). Alterations of the tumor suppressor genes CDKN2A (p16(INK4a)), p14(ARF), CDKN2B (p15(INK4b)), and CDKN2C $(\mathrm{p} 18(\mathrm{INK} 4 \mathrm{c}))$ in atypical and anaplastic meningiomas Am J Pathol (Vol. 159, pp. 661-669). United States.

Bujko, M., Kober, P., Tysarowski, A., Matyja, E., Mandat, T., Bonicki, W., \& Siedlecki, J. A. (2014). EGFR, PIK3CA, KRAS and BRAF mutations in meningiomas. Oncol Lett, 7(6), 2019-2022. doi: 10.3892/ol.2014.2042

Choy, W., Kim, W., Nagasawa, D., Stramotas, S., Yew, A., Gopen, Q., . . . Yang, I. (2011). The molecular genetics and tumor pathogenesis of meningiomas and the future directions of meningioma treatments. Neurosurg Focus, 30(5), E6. doi: 10.3171/2011.2.focus1116

Duverger, A., Wolschendorf, F., Anderson, J. C., Wagner, F., Bosque, A., Shishido, T., .. Kutsch, O. (2013). Kinase control of Latent HIV-1 Infection: PIM-1 Kinase as a Major Contributor to HIV-1 Reactivation. $J$ Virol. doi: 10.1128/JVI.02682-13

Fang, Z., Grutter, C., \& Rauh, D. (2013). Strategies for the selective regulation of kinases with allosteric modulators: exploiting exclusive structural features. ACS Chem Biol, 8(1), 58-70. doi: 10.1021/cb300663j

Golubovskaya, V. M. (2014). Targeting FAK in human cancer: from finding to first clinical trials. Front Biosci (Landmark Ed), 19, 687-706.

Graham, D. K., DeRyckere, D., Davies, K. D., \& Earp, H. S. (2014). The TAM family: phosphatidylserine sensing receptor tyrosine kinases gone awry in cancer. Nat Rev Cancer, 14(12), 769-785.

James, M. F., Han, S., Polizzano, C., 
Plotkin, S. R., Manning, B. D., StemmerRachamimov, A. O., . . . Ramesh, V. (2009). NF2/merlin is a novel negative regulator of mTOR complex 1, and activation of mTORC1 is associated with meningioma and schwannoma growth $\mathrm{Mol}$ Cell Biol (Vol. 29, pp. 4250-4261). United States.

Jarboe, J. S., Dutta, S., Velu, S. E., \& Willey, C. D. (2013). Mini-review: bmx kinase inhibitors for cancer therapy. Recent Pat Anticancer Drug Discov, 8(3), 228238.

Jenkinson, M. D., Weber, D. C., Haylock, B. J., Mallucci, C. L., Zakaria, R., \& Javadpour, M. (2015). Atypical meningoma: current management dilemmas and prospective clinical trials. $J$ Neurooncol, 121(1), 1-7. doi: 10.1007/s11060-014-1620-1

Kaley, T. J., Wen, P., Schiff, D., Ligon, K., Haidar, S., Karimi, S., . . . Omuro, A. (2015). Phase II trial of sunitinib for recurrent and progressive atypical and anaplastic meningioma. Neuro Oncol, 17(1), 116-121. doi: 10.1093/neuonc/nou148

Komotar, R. J., Iorgulescu, J. B., Raper, D. M., Holland, E. C., Beal, K., Bilsky, M. H., . . Gutin, P. H. (2012). The role of radiotherapy following gross-total resection of atypical meningiomas. $J$ Neurosurg, 117(4), 679-686. doi: 10.3171/2012.7.JNS112113

Mawrin, C., Sasse, T., Kirches, E., Kropf, S., Schneider, T., Grimm, C., . . . Dietzmann, K. (2005). Different activation of mitogen-activated protein kinase and Akt signaling is associated with aggressive phenotype of human meningiomas Clin Cancer Res (Vol. 11, pp. 4074-4082). United States.

Norden, A. D., Drappatz, J., \& Wen, P. Y. (2007). Targeted drug therapy for meningiomas. Neurosurg Focus, 23(4), E12. doi: 10.3171/FOC-07/10/E12

Powers, A. D., \& Palecek, S. P. (2012). Protein analytical assays for diagnosing, monitoring, and choosing treatment for cancer patients. J Healthc Eng, 3(4), 503-534. doi: 10.1260/20402295.3.4.503

Sikkema, A. H., Diks, S. H., den Dunnen, W. F., ter Elst, A., Scherpen, F. J., Hoving, E. W., . . . de Bont, E. S. (2009). Kinome profiling in pediatric brain tumors as a new approach for target discovery Cancer Res (Vol. 69, pp. 5987-5995). United States.

Simpson, D. (1957). The recurrence of intracranial meningiomas after surgical treatment. J Neurol Neurosurg Psychiatry, 20(1), 22-39.

Wang, Q., Zorn, J. A., \& Kuriyan, J. (2014). A structural atlas of kinases inhibited by clinically approved drugs. Methods Enzymol, 548, 23-67. doi: 10.1016/B978-0-12-397918-6.00002-1 